\title{
Is the Broad-Line Region Expanding? ${ }^{1}$
}

Kan Liang and Xiang-Tao He

Department of Astronomy, Beijing Normal University, Beijing 100875, China

Zhong-min Wang and Jun-Han You

Institute for Space Astrophysics, Department of Applied Physics, Shanghai Jiao Tong University, Shanghai 200030, China

\section{Introduction}

We suggest that the broad-line regions (BLRs) of QSOs with broad emissionlines (BALs) are expanding, i.e., the clouds are undergoing radial outflow, as illustrated simply in Fig. 1. At least the following observational facts can be explained:

1. Optical observations show that some moderate and high-redshift QSOs have BALs. The number of these QSOs is about $3-10 \%$ of all QSOs (Foltz et al. 1990). One can see in Fig. 2 that very low-redshift QSOs have no BALs.

2. Almost all BAL QSOs exhibit $z_{a b s}<z_{e m}$.

3. High-resolution observations reveal that the widths of the broad absorption lines are narrower than that of the corresponding emission line for all $\mathrm{BAL}$ QSOs, i.e. $\Delta \lambda_{a b}<\Delta \lambda_{e m}$.

\section{Discussion and Conclusion}

All these facts can be easily understood if the BLR of QSOs with BALs is expanding. Firstly, high-redshift QSOs were formed in the early Universe at which time the expansion of the BLR had just begun, and therefore the covering factor $C$ is large, and the absorption lines are easily formed. In the process of evolution, as the covering factor $C$ decreases, absorption lines disappear gradually while emission lines persist. This is consistent with the redshift distribution BAL QSOs (see Fig. 2).

Secondly, one can only receive the line radiation from the clouds whose ionized layer is facing towards the observer. Therefore a Doppler redshift of the emission lines will be expected (see Fig. 1). However, the absorption lines come from those clouds which are located between the continuum source and the

\footnotetext{
${ }^{1}$ This work is supported by the National Natural Science Foundation of China.
} 


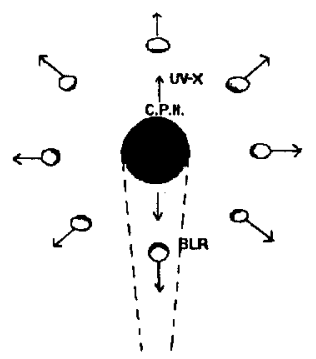

Figure 1. A schematic view of the BLR with clouds in outward radial motion.

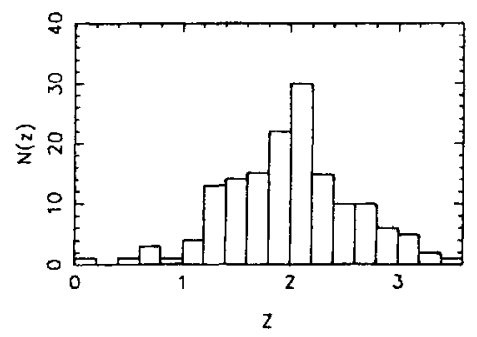

Figure 2. The distribution in redshift $z$ of BAL QSOs.

observers, so a blueshift of absorption lines will be expected due to the expansion of clouds (Fig. 1).

Thirdly, the narrower absorption-line widths can be easily explained in our outflow model of the BLR. The absorption lines can be formed only when the absorption clouds enter into the solid angle subtended at the observer by the source of continuum, so the absorption line is narrower than the corresponding emission line due to the fact that the number of the absorption clouds is very small.

Upon explaining the above observational facts, we believe that our expanding BLR model is reasonable.

\section{References}

Foltz, C. B., et al. 1990, BAAS, 22, 806.

Hazard, C., et al. 1984, ApJ, 282, 33.

Hewitt, A., \& Burbidge, G. 1993 ApJS, 87.

Sargent, W.L.W., et al. 1989 ApJS, 69, 703.

Turnshek, D. A. 1986, in QSO Absorption Lines, ed. J. C. Blades, D. Turnshek, and C.A. Norman, (Cambridge: Cambridge Univ. Press), p. 17. 